\title{
沉淀聚合制备诺氟沙星 $-\mathrm{Zn}^{2+}$ 分子印迹聚合物及其印迹方式的研究
}

\author{
孙妍王兵* \\ (天津工业大学中空纤维膜材料与膜过程省部共建国家重点实验室培育基地, \\ 天津工业大学环境与化学工程学院 天津 300160)
}

\begin{abstract}
摘要 采用沉淀聚合的方式以诺氟沙星(NFA)- $\mathrm{Zn}^{2+}$ 为模板分子, 乙二醇二甲基丙烯酸酯为交联剂, 分别选取酸性功能 单体甲基丙烯酸与碱性功能单体 4-乙烯基吡啶制备了诺氟沙星 $-\mathrm{Zn}^{2+}$ 的分子印迹聚合物. 通过紫外光谱研究发现诺氟沙 星与 $\mathrm{Zn}^{2+}$ 及两种功能单体均发生了金属配位印迹作用且形成了比例不同的印迹复合物. 红外光谱的功能基团的表征结 果显示, 甲基丙烯酸与诺氟沙星- $\mathrm{Zn}^{2+}$ 形成了以诱导作用占优的三元配合物, 而 4-乙烯基吡啶则与诺氟沙星- $\mathrm{Zn}^{2+}$ 形成 了共轭作用占优的三元配合物. 扫描电镜及粒径分布实验表征了聚合物的物理特性, 结果显示印迹聚合物的表面存在 孔及孔道结构而非印迹聚合物的表面较致密不存在孔且制备的印迹聚合物的粒径均在 $100 \mu \mathrm{m}$ 以下, 其平均粒径为 39 $\mu \mathrm{m}$. 等温结合及选择性实验的结果表明 4-乙烯基吡啶为功能单体制备的印迹聚合物的选择性识别性能优于甲基丙烯 酸为功能单体制备的印迹聚合物, 其特异性吸附容量和印迹指数分别为 $66.84 \mu \mathrm{mol} / \mathrm{g}$ 和 4.207. 同时在混合溶液的选择 性实验中以 4-乙烯基吡啶(4-VP)为功能单体制备的印迹聚合物的选择识别诺氟沙星的能力优于以甲基丙烯酸为功能单 体的聚合物, 其识别因子分别为 3.408 和 2.909 , 而非印迹聚合物对底物的吸附量较小且识别因子均接近于 1 , 说明非印 迹聚合物对底物的识别为非选择性的.
\end{abstract}

关键词＼cjkstart诺氟沙星- $\mathrm{Zn}^{2+}$; 沉淀聚合; 金属配位; 诱导作用; 共轭作用

\section{Synthesis of Norfloxacin-Zinc (II) Imprinted Polymer by Precipitation Polymerization and Study on Its Imprinting Form}

\author{
Sun, Yan Wang, Bing* \\ (State Key Laboratory of Hollow Fiber Membrane Materials and Processes, Tianjin Polytechnic University, School of Envi- \\ ronmental and Chemical Engineering, Tianjin Polytechnic University, Tianjin 300160)
}

\begin{abstract}
A group of molecularly imprinted polymer (MIP) with specific molecule identification properties for norfloxacin (NFA) was prepared by precipitation polymerization using norfloxacin- $\mathrm{Zn}^{2+}$ as template molecule, ethyleneglycol dimethacrylant as the cross-linker, methacrylic acid and 4-vinyl pyridine as functional monomer respectively. The coordination proportion of the template molecule and functional monomer was studied by ultraviolet spectrum and the characterization of the molecularly imprinted polymer can be achieved by the infrared spectrum analysis. The result indicated that dominant induction imprinted complex with the ratio $1: 1$ was formed between norfloxacin- $\mathrm{Zn}^{2+}$ and methacrylic acid, while the dominant conjugation imprinted complex was formed by 4-vinyl pyridine as the functional monomer with the ratio of $1: 2$, and the ChemDraw pictures were used to display the imprinted form of the polymers. Then the scanning electron microscopy and the particle size distribution were used to characterize the physical properties of the molecularly imprinted polymer, the result showed irregular holes and porous scattered in the prepared molecularly imprinted polymer and the non-imprinted polymer surface was dense with no holes, then the particle size of the prepared polymer was less than $100 \mu \mathrm{m}$ and the average particle size was $39 \mu \mathrm{m}$. The adsorption ability of the prepared polymer were researched by the isothermal combined experiment and the result revealed that the prepared molecularly imprinted polymer using 4-vinyl pyridine with the ring space structure as the functional monomer showed better selectivity recognition ability for norfloxacin than methacrylic acid with the chain structure, the specificity adsorption was $66.84 \mu \mathrm{mol} / \mathrm{g}$ and the imprinted index was 4.207, and it also showed better selective ability in the nofloxacin- $\mathrm{Zn}^{2+}$ and ofloxacin- $\mathrm{Zn}^{2+}$ mixture solution, the recognition factor was 3.408. Meanwhile the selective experiment displayed that the adsorption capacity of the non-imprinted polymer was small and closed to each other, therefore the recognition factor was close to 1 , this indicated that the recognition ability of the non-imprinted polymer to the adsorption substrate was non selective.

Keywords norfloxacin- $\mathrm{Zn}^{2+}$; precipitation polymerization; metal coordination; induction; conjugation
\end{abstract}

* E-mail: bingwang666@yahoo.com.cn

Received March 28, 2012; published May 23, 2012.

Supporting information for this article is available free of charge via the Internet at http://sioc-journal.cn

Project supported by the Natural Science Foundation of Tianjin (No. 10JCZDJC21900).

项目受天津市自然科学基金重点项目(No. 10JCZDJC21900)资助. 


\section{1 引言}

分子印迹技术是一种具有分子特异性识别能力的 新型分离技术 ${ }^{[1 \sim 3]}$, 基于印迹技术制备的印迹聚合物在 诸多领域具有潜在的应用价值 ${ }^{[4 ~ 7]}$. 传统的印迹聚合物 的制备多采用本体聚合或溶液聚合, 但后处理过程繁琐 且印迹聚合物的收率较低 ${ }^{[8,9]}$. 沉淀聚合是一种优于本 体聚合的制备方法, 其优点在于避免了本体聚合中聚合 物粉碎过篎时的印迹位点的破坏及聚合物的浪费, 且制 得的聚合物粒径较均一，印迹孔穴多暴露在聚合物表 面 ${ }^{[10 ~ 12]}$. 同时人们在研究生物识别过程中的相互作用 力时发现生物或者药物分子和金属离子的结合具有高 度专一性及温和的结合和断裂条件 ${ }^{[13,14]}$. 即金属离子配 位作用具有强度优于传统印迹聚合物中的非共价键作 用力，且在极性溶剂体系中较稳定等优势.

目前应用金属离子配位作用制备分子印迹聚合物 的报道较多，且制备的金属印迹聚合物得到了多方面的 广泛应用, 但对金属印迹作用机理的研究较少. 特别是 制备金属印迹聚合物时功能单体的选择及其对印迹方 式的影响 ${ }^{[15,16]}$. 本文选取易与金属配位的诺氟沙星 (NFA)为研究对象, 甲基丙烯酸(MAA)和 4-乙烯基吡啶 (4-VP) 分别为功能单体, 在沉淀聚合法制备印迹聚合物 的同时研究功能单体对印迹方式的影响. 由于诺氟沙星 为第二代喹诺酮类抗菌药 ${ }^{[17]}$, 其学名为 1-乙基-6 氟-1,4二氢-4-氧代-7-(1-哌嗪基)喹啉-3-羧酸(图 1), 通过作用 于消化道内致病细菌 DNA 拓扑异构酶, 抑制细菌 DNA 的正常合成和复制而导致细菌死亡, 具有广谱抗菌作 用, 故大量广泛地用于人体临床和畜禽养殖, 但 $30 \%$ 左 右是通过原形排出体外进入环境, 而 $15 \%$ 是以代谢物的 形式从尿液中排出, 对生态环境具有潜在的不良影响且 制备的印迹聚合物在诺氟沙星的环境检测及富集方面 具有潜在应用前景 ${ }^{[18,19]}$.

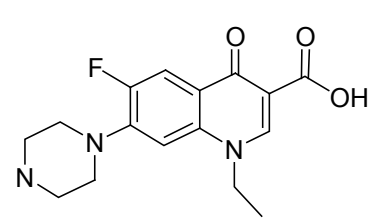

(a)

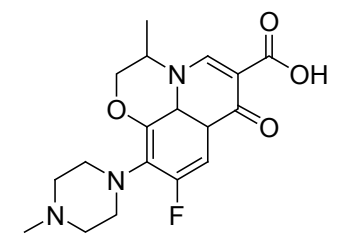

(b)
图 1 诺氟沙星和氧氟沙星的化学结构式

Figure 1 Chemical structure of norfloxacin and ofloxacin

\section{2 结果与讨论}

\section{1 紫外结果分析}

\section{1 .1 作用力分析}

在弱酸性条件下 NFA 有两个峰, $272 \mathrm{~nm}$ 处的单峰, $322 \mathrm{~nm}$ 处的肩峰. $272 \mathrm{~nm}$ 处为 $\pi-\pi^{*}$ (A 环)归属于苯环连 接生色团 $N$ 杂环与助色团杂原子 $F$ 产生. $322 \mathrm{~nm}$ 处肩峰
为 $\pi-\pi^{*}$ (B 环)归属于苯酰基化合物 $\mathrm{K}$ 带. 在 NFA 结构中 $\mathrm{C}=\mathrm{O}, \mathrm{COOH}$ 及哌嗪基上的 $\mathrm{N}$ 原子都可以作为电子供 体易参与金属配位 ${ }^{[20]}$. 而哌嗪基上的 $\mathrm{N}$ 原子由于在弱酸 性溶液中被质子化而不易参与金属配位. 保持 NFA 的 浓度不变, 逐渐增大 $\mathrm{Zn}^{2+}$ 的浓度(图 2a 2d) $272 \mathrm{~nm}$ 单 峰红移至 $276 \mathrm{~nm}, 322 \mathrm{~nm}$ 处的峰值不断蓝移且两峰发生 增色效应. 说明超分子体系的形成, 即 NFA 与金属离子 发生了配位作用. 同时在溶液 $n(\mathrm{NFA}): n\left(\mathrm{Zn}^{2+}\right)=1: 1$ 加入不同功能单体后谱图发生较大的变化, 说明加入的 功能单体与模板金属配合物发生了较强的作用, 图 3 中 $\mathrm{A}$ 与 $\mathrm{C}$ 谱线分别为功能单体 $\mathrm{MAA}$ 与 4-VP, $\mathrm{B}$ 与 $\mathrm{D}$ 谱线 则为分别加入模板金属配合物的 MAA 与 4-VP 变化的 谱线. 对比 $\mathrm{A}$ 与 $\mathrm{B}$ 谱线发现 $218 \mathrm{~nm}$ 处的 $\mathrm{n}-\pi^{*}$ *跃迁红移 至 $224 \mathrm{~nm}$ 并伴随吸光度下降而在 $306 \mathrm{~nm}$ 处出现了新的 吸收峰, 说明功能单体与模板金属配合物之间发生了配 位作用, 配位作用使共轭体系延长, 使共轭体系中电子 云密度降低, 同时增加了 $\pi$ 电子的流动性, 从而 $n-\pi *$ 跃 迁发生了小幅红移. 同时配合物的 $\pi$ 轨道耦合后因 $\pi$ 电 子的流动性填充, 使跃迁几率减小, 产生减色效应. 再 对比谱线 C与 D $205 \mathrm{~nm}$ 处及 $242 \mathrm{~nm}$ 处的峰都发生了较 大幅度的红移及减色效应, 特别是 $242 \mathrm{~nm}$ 处的峰红移 至 $278 \mathrm{~nm}$ 处且吸光度减小幅度较大. 这是由于形成复 合物所结合的 4-乙烯基吡啶增加了诺氟沙星分子中苯 环周围的空间位阻, 使其电子跃迁几率减小, 吸光度也 相应降低. 两功能单体吸收峰位的改变说明诺氟沙 星 $-\mathrm{Zn}^{2+}$ 与两功能单体分别发生了配合作用 ${ }^{[21]}$.

\section{1 .2 配合比例分析}

设模板分子 $(\mathrm{T})$ 浓度为 $a_{0}$, 功能单体 $(\mathrm{M})$ 浓度 $b_{0}$, 化 学配位比为 $n$, 则结合反应如下 ${ }^{[22]}$ :

$$
\begin{aligned}
& \mathrm{T}+n \mathrm{M} \rightarrow \mathrm{T}[\mathrm{M}]_{n} \\
& K=\left[\mathrm{T}[\mathrm{M}]_{n}\right] /[\mathrm{T}][\mathrm{M}]^{n}
\end{aligned}
$$

根据物料平衡

$$
\begin{aligned}
& {[\mathrm{T}]+\left[\mathrm{T}[\mathrm{M}]_{n}\right]=a_{0}} \\
& {[\mathrm{M}]+\left[\mathrm{T}[\mathrm{M}]_{n}\right]=b_{0}}
\end{aligned}
$$

因为 $b_{0}$ 远大于 $a_{0}$, 可忽略 $\left[\mathrm{T}[\mathrm{M}]_{n}\right]$, 所以

$$
[\mathrm{M}] \approx b_{0}
$$

将(3)和(4)带入(2)得

$$
\left[\mathrm{T}[\mathrm{M}]_{n}\right]=a_{0} b_{0}{ }^{n} K /\left(1+b_{0}{ }^{n} K\right)
$$

根据 Lamber-Beer 定律, 若溶液中只有 $\mathrm{T}$ 和 $\mathrm{T}[\mathrm{M}]_{n}$ 有吸收, 设 $\varepsilon_{1}$ 和 $\varepsilon_{2}$ 分别为 $\mathrm{T}$ 和配合物的摩尔吸收系数, 则总吸光度为

$$
\begin{gathered}
A=\varepsilon_{1} l[\mathrm{~T}]+\varepsilon_{2} l\left[\mathrm{~T}[\mathrm{M}]_{n}\right]=\varepsilon_{1} l\left(a_{0}-\left[\mathrm{T}[\mathrm{M}]_{n}\right]\right)+ \\
\varepsilon_{2} l\left[\mathrm{~T}[\mathrm{M}]_{n}\right]=\left(\varepsilon_{2}-\varepsilon_{1}\right) l\left[\mathrm{~T}[\mathrm{M}]_{n}\right]+\varepsilon_{1} l a_{0} \\
\text { 令 } A_{0}=\varepsilon_{1} l a_{0}, \text { 则吸光度的变化为 } \\
\Delta A=A-A_{0}=\left(\varepsilon_{2}-\varepsilon_{1}\right) l\left[\mathrm{~T}[\mathrm{M}]_{n}\right]=\Delta \varepsilon l\left[\mathrm{~T}[\mathrm{M}]_{n}\right]
\end{gathered}
$$

将(7)带入(5)得:

$$
\Delta A / b_{0}{ }^{n}=-K \Delta A+K \Delta \varepsilon l a_{0}
$$


化学配位比 $n$ 分别取 1, 2, 3 时, 以 $\Delta A / b_{0}{ }^{n}$ 对 $\Delta A$ 作 图, NFA- $\mathrm{Zn}^{2+}$ 为客体, 4-VP 为主体, 当 $n=2$ 时 $\Delta A / b_{0}{ }^{n}$ 与 $\Delta A$ 有线性函数关系(图 4A), 回归方程为: $\Delta A / b_{0}{ }^{n}=$ $-0.0501 \Delta A+0.0191, r=0.9974$. NFA- $\mathrm{Zn}^{2+}$ 为客体, MAA 为主体, 当 $n=1$ 时 $\Delta A / b_{0}{ }^{n}$ 与 $\Delta A$ 有线性函数关系

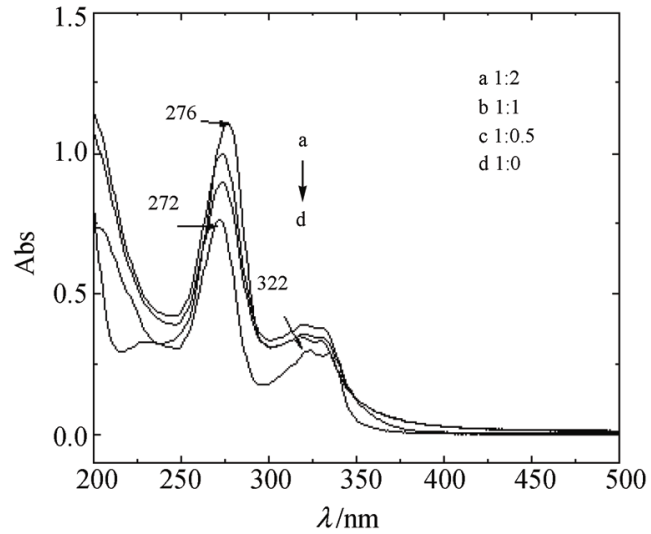

图 2 乙腈溶液中诺氟沙星与 $\mathrm{Zn}^{2+}$ 在不同物质的量之比时的紫外谱图 Figure 2 UV-Vis spectra of norfloxacin and $\mathrm{Zn}^{2+}$ with different molar ratios in acetonitrile

(a) $1: 2$; (b) $1: 1 ;$ (c) $1: 0.5 ;$ (d) $1: 0$

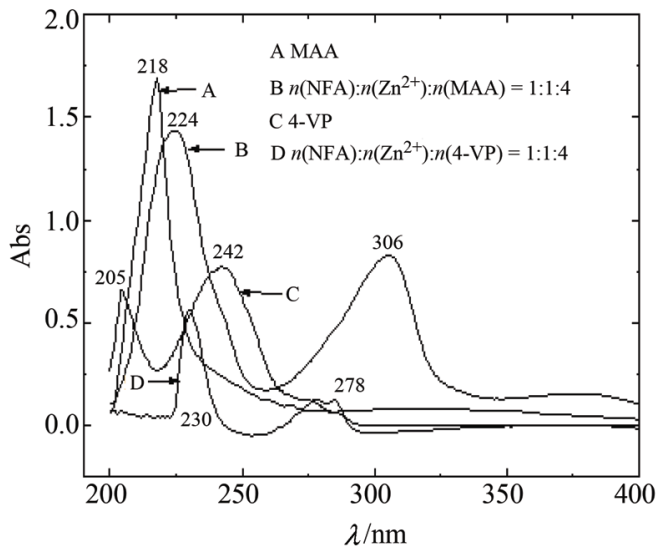

图 3 模板分子与功能单体的紫外谱图

Figure 3 UV-vis spectra of monomer and complexes

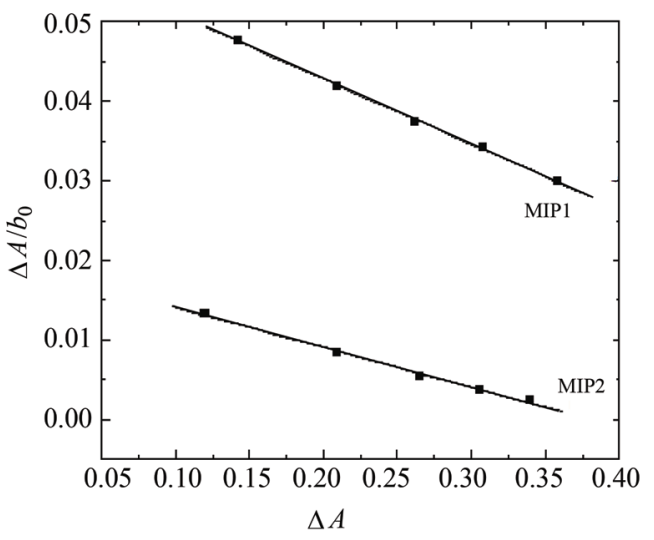

图 4 印迹聚合物的配位方程

Figure 4 Line of coordination equation of MIP

The functional monomer of $\mathrm{MIP}_{1}$ and $\mathrm{MIP}_{2}$ were methacrylic-acid and 4-vinyl pyridine, respectively
(图 4B), 回归方程: $\Delta A / b_{0}{ }^{n}=-0.0819 \Delta A+0.0592$.

以上说明在聚合反应中，模板分子分别与 4-VP 及 MAA 形成 $1: 2$ 及 $1: 1$ 型配合物, 其复合物的结合常 数 $K$ 分别为 $5.01 \times 10^{4} \mathrm{~L}^{2} \cdot \mathrm{mol}^{-2}$ 和 $8.19 \times 10^{4} \mathrm{~L}^{2} \cdot \mathrm{mol}^{-2}$.

\section{2 红外光谱分析}

红外光谱是一种较直接的表征金属配合作用形成 的谱学手段之一，金属配合作用会影响模板分子中的官 能团的振动, 故对比模板分子及印迹聚合物可以推测进 行金属印迹的官能团 ${ }^{[23]}$. 图 5 中的三条曲线分别为诺氟 沙星(a), $\mathrm{MIP}_{2}$ (b), MIP $(\mathrm{c})$, 都存在 $3300 \sim 3500 \mathrm{~cm}^{-1}$ 的 $\mathrm{O}-\mathrm{H}$ 与 $\mathrm{N}-\mathrm{H}$ 的伸缩振动叠加峰, $3000 \mathrm{~cm}^{-1}$ 左右的 $\mathrm{Ar}-\mathrm{H}$ 的伸缩振动谱带, 及 $2500,1460,1265,1150,1033$ $\mathrm{cm}^{-1}$ 分别归属于 $\mathrm{C}=\mathrm{N}$ 、芳环骨架、 $\mathrm{C}-\mathrm{N} 、 \mathrm{C}-\mathrm{F}$ 取代 伸缩振动是 NFA 的特征官能团. 同时谱线 $\mathrm{b}$ 中存在 4-VP 芳香环的骨架振动峰 1605,1585 和 $1477 \mathrm{~cm}^{-1}$, 而 谱线 $\mathrm{c}$ 中存在 $1454 \mathrm{~cm}^{-1} \mathrm{MAA}$ 中甲基的变形振动峰, 说 明模板分子与功能单体成功地进行了配位印迹作用.

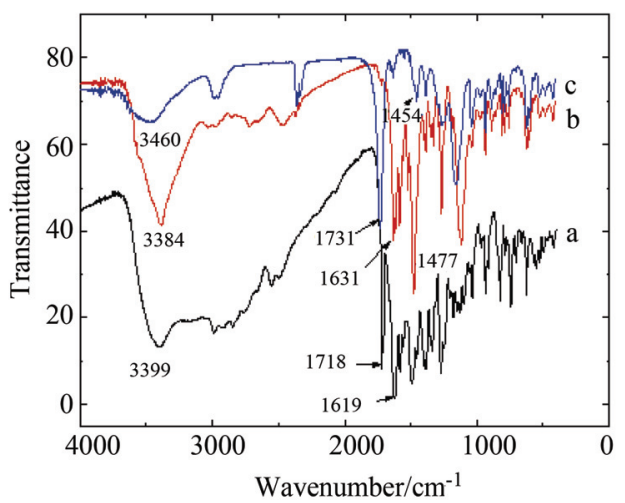

图 5 诺氟沙星(a), $\mathrm{MIP}_{2}$ (b) 和 $\mathrm{MIP}_{1}$ (c) 的红外光谱

Figure 5 Infrared spectra of norfloxacin (a), $\mathrm{MIP}_{2}$ (b) and $\mathrm{MIP}_{1}$ (c) The functional monomer of $\mathrm{MIP}_{1}$ and $\mathrm{MIP}_{2}$ were methacrylic-acid and 4-vinyl pyridine, respectively

根据谱线 $\mathrm{b}, \mathrm{c}$ 的变化可知在与金属及功能单体发生 配位作用后 NFA 中的 $\mathrm{C}=\mathrm{O}$ 的伸缩振动频率变化最为 显著. 由于 NFA 中的两个 $\mathrm{C}=\mathrm{O}$ 是同类官能团(谱线 $\mathrm{a}$ ), 且在结构中彼此接近，它们的振动频率发生了干扰，产 生了吸收峰相差较大的情况, 即 1718 与 $1619 \mathrm{~cm}^{-1}$ 处的 两个吸收峰, 其中高频谱带相应于两个 $\mathrm{C}=\mathrm{O}$ 的不对称 伸缩振动，低频的谱带则相当于对称伸缩振动. 其综合 峰位应在 $1670 \mathrm{~cm}^{-1}$ 左右. 在发生配位作用后, 两吸收 峰变为单峰且发生了不同程度的移动, 即 $\mathrm{b}$ 中 $\mathrm{C}=\mathrm{O}$ 峰 位红移至 $1631 \mathrm{~cm}^{-1}$, 而 $\mathrm{c}$ 中则蓝移至 $1731 \mathrm{~cm}^{-1}$. 说明 4-VP 和 MAA 与 NFA 的配位方式不同. $\mathrm{NFA}-\mathrm{Zn}^{2+}$ 与 4-VP 配位后, 使得 NFA 中 $\mathrm{C}=\mathrm{O}$ 的电子离域发生改变, 且一个 $\mathrm{Zn}^{2+}$ 离子需要两个 4-VP 中的 $\mathrm{N}$ 原子进行配位, 4-VP 的吡啶环在一定程度上使得共轭效应占优，并通 过 $\pi$ 键的传递, 引起双键的极性增加, 双键性降低, 因 而使 $\mathrm{C}=\mathrm{O}$ 的伸缩振动频率下降, 产生了红移现象, 同 
时吡啶环也增强了 NFA 的场效应从而增强了红移幅度. 而与 MAA 作用时, MAA 中的 $\mathrm{O}$ 原子的电负性较大, 且 MAA 的共轭结构简单, 使得诱导效应占优, 并沿着分 子中的 $\sigma$ 键传递, 引起 $\mathrm{C}=\mathrm{O}$ 氧原子上的电荷相对贫乏,
$\mathrm{C}=\mathrm{O}$ 键的伸缩振动能升高, $\mathrm{C}=\mathrm{O}$ 峰波数增加, 产生了 蓝移现象. 根据 NFA 的结构推测印迹方式如图 6 和图 7.<smiles>CCn1cc(C(=O)O)c(=O)c2cc(F)c(N3CCNCC3)cc21</smiles>

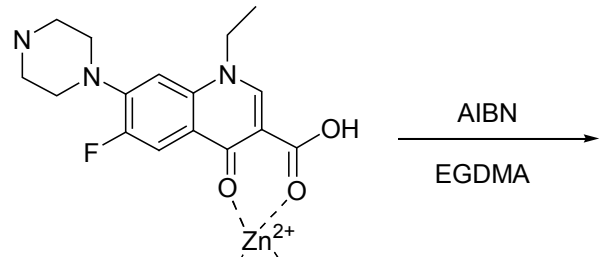
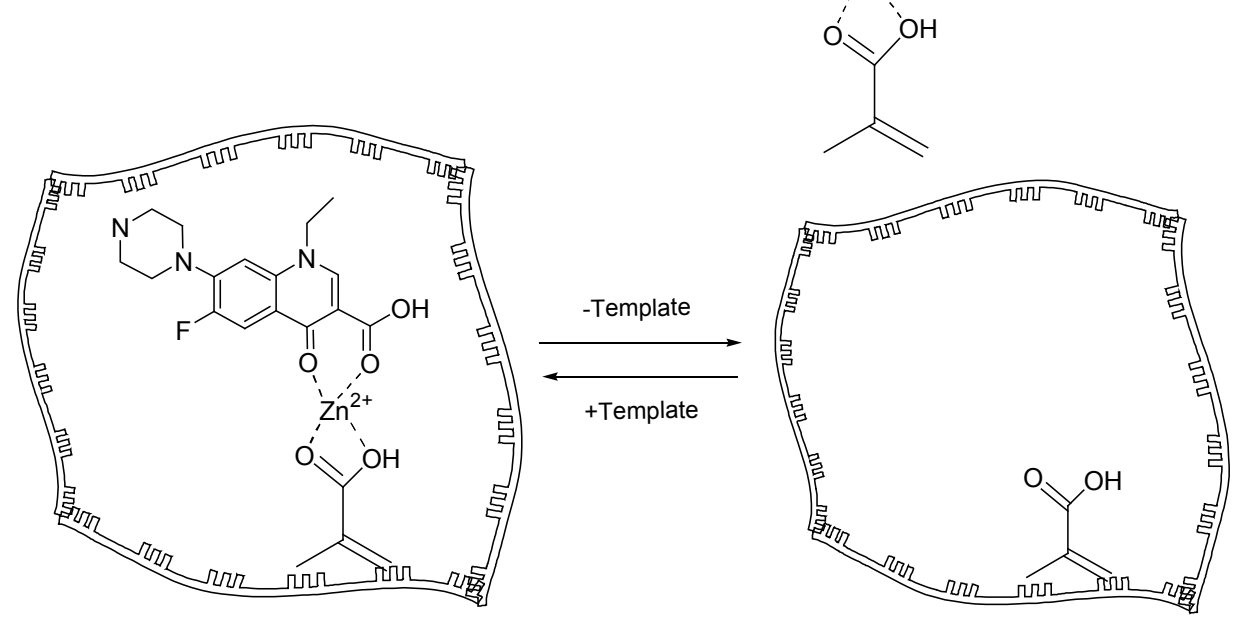

图 $6 \mathrm{NFA}-\mathrm{Zn}^{2+}$ 和 MAA 的印迹作用图

Figure 6 Schematic diagram of NFA- $\mathrm{Zn}^{2+}$ and MAA

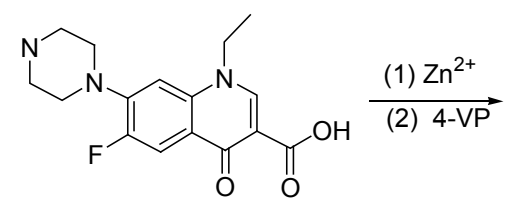<smiles>CCn1cc(C(=O)O)c(=O)c2cc(F)c(N3CCNCC3)cc21</smiles>
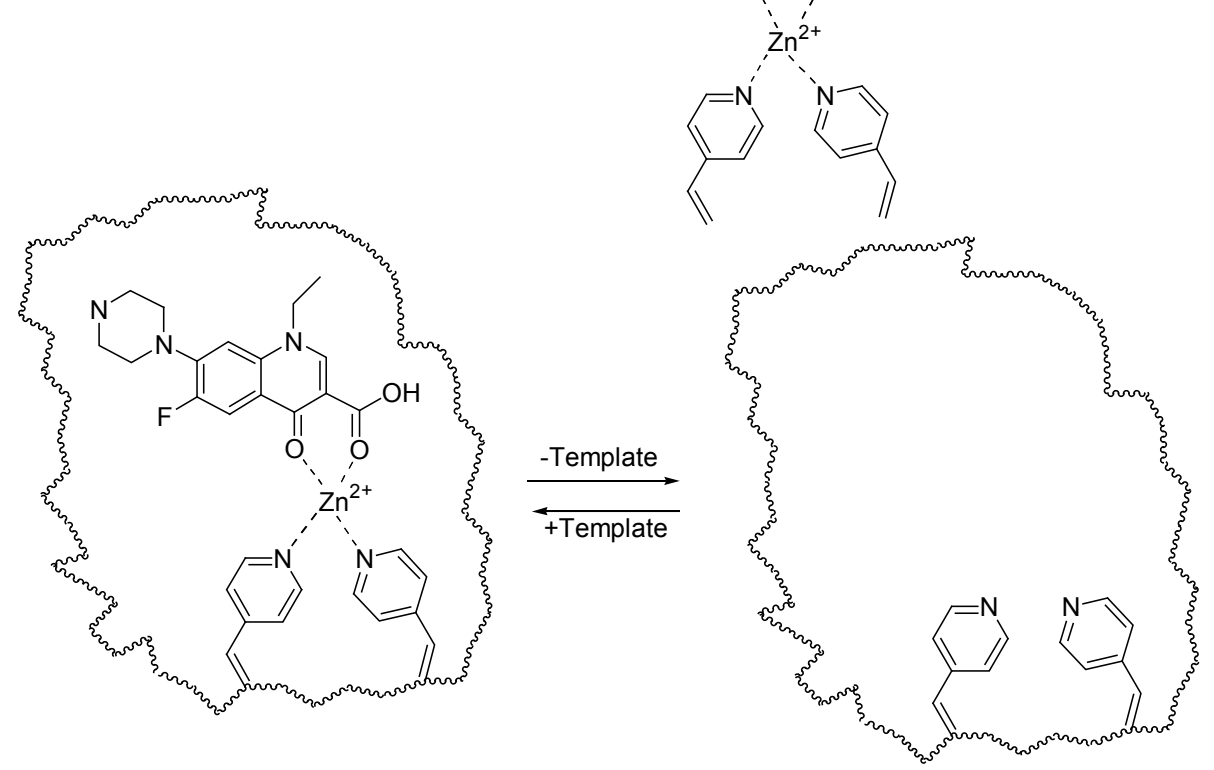

图 7 NFA- $\mathrm{Zn}^{2+}$ 和 4-VP 的印迹作用图

Figure 7 Schematic diagram of NFA- $\mathrm{Zn}^{2+}$ and 4-VP 


\section{3 电镜及粒径分布}

利用 SEM 可以直接观察聚合物表面表观形态、孔 分布等. 由扫描电镜图(图 8)对比 $\mathrm{A}$ 与 $\mathrm{B}$ 发现在印迹聚 合物的表面存在很多大小不一的孔, 而非印迹聚合物的 表面较平滑, 且不含有交错的孔洞结构. 这也说明制备 的印迹聚合物是一种多孔的交联聚合物, 由于模板分子 的加入形成了多孔结构, 这些结构为溶质的扩散和质量 的交换提供了良好的场所和通道. 同时根据粒径分布曲 线可知(图 9), 制备的聚合物的粒径均在 $100 \mu \mathrm{m}$ 以内, 平均粒径为 $39 \mu \mathrm{m}$.
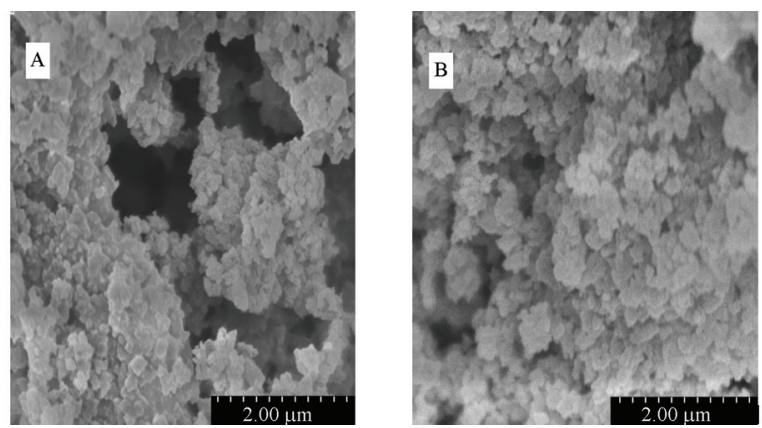

图 8 印迹聚合物 $\mathrm{MIP}_{2}(\mathrm{~A})$ 及非印迹聚合物 $\mathrm{NIP}_{2}(\mathrm{~B})$ 的扫描电镜图 Figure 8 SEM picture of $\mathrm{MIP}_{2}(\mathrm{~A})$ and $\mathrm{NIP}_{2}(\mathrm{~B})$

The functional monomer of $\mathrm{MIP}_{2}$ was $4-\mathrm{VP}$

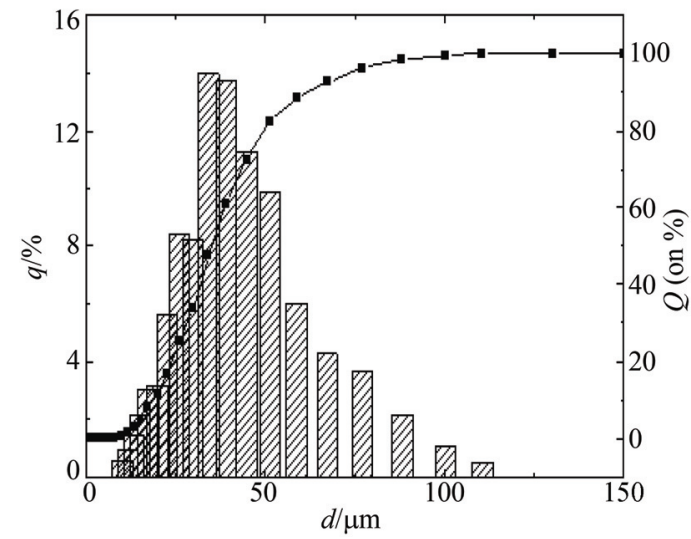

图 9 印迹聚合物 $\mathrm{MIP}_{2}$ 的粒径分布图

Figure 9 Particle size distributions of $\mathrm{MIP}_{2}$ particles

The functional monomer of $\mathrm{MIP}_{2}$ was 4-vinyl pyridine

\section{4 聚合物吸附性能研究}

\section{4 .1 等温结合曲线}

测定聚合物的平衡吸附量 $Q$ 随着 NFA 初始浓度的 变化 $(0.05 \sim 2.5 \mathrm{mmol} / \mathrm{L})$, 如图 10 所示在研究浓度范围 内, $\mathrm{MIP}_{1}$ 与 $\mathrm{MIP}_{2}$ 的吸附曲线都基本符合朗格缪单分子 层吸附模型, $\mathrm{NIP}_{1}$ 与 $\mathrm{NIP}_{2}$ 的吸附曲线线性较高, 且 $\mathrm{MIP}_{1}$ 及 $\mathrm{NIP}_{1}$ 的单点吸附量均略高于 $\mathrm{MIP}_{2}$ 及 $\mathrm{NIP}_{2}$. 说明印迹 识别位点基本暴露在聚合物的表面, 随着初始浓度的增 大印迹聚合物对 NFA-Zn ${ }^{2+}$ 的吸附量均增加, 但 MIP $_{1}$ 的 结合量总是略大于 $\mathrm{MIP}_{2}$, 表现出更优越的结合性能. 而 非印迹聚合物的结合曲线线性增加, 难于达到饱和, 即

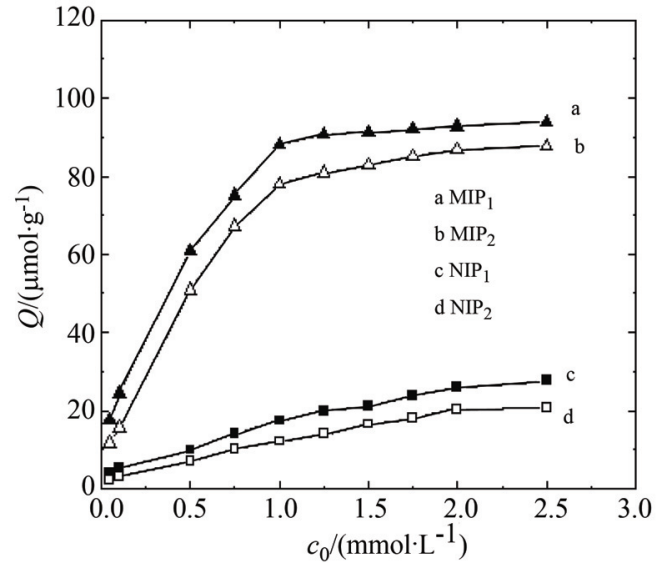

图 10 印迹聚合物及非印迹聚合物的等温结合曲线

Figure 10 Adsorption curve of MIP and NIP under different initial concentration

$\mathrm{NIP}_{1}$ 与 $\mathrm{NIP}_{2}$ 对 NFA-Zn ${ }^{2+}$ 为非选择性吸附. 特异性吸附 容量 $Q^{\prime}$ 和印迹指数 $B$ 的计算 ${ }^{[24]}: Q^{\prime}=Q_{\mathrm{M}}-Q_{\mathrm{N}}, B=$ $Q_{\mathrm{M}} / Q_{\mathrm{N}}$, 式中 $Q_{\mathrm{M}}$ 和 $Q_{\mathrm{N}}$ 分别是 MIP 和 NIP 在相同条件下 对模板分子的吸附量. $\mathrm{MIP}_{1}$ 的特异性吸附量 $Q^{\prime}=65.97$ $\mu \mathrm{mol} / \mathrm{g}$, 印迹指数为 3.380. $\mathrm{MIP}_{2}$ 的特异性吸附量 $Q^{\prime}=$ $66.84 \mu \mathrm{mol} / \mathrm{g}$, 印迹指数为 4.207 .

在分子印迹聚合物的制备过程中，预组织复合物的 有效固定量及复合物固定时形成的特定的三维空间结 构, 直接影响再识别过程中印迹聚合物对模板分子的结

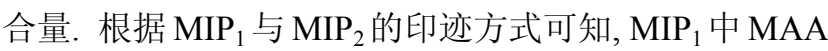
与 NFA- $\mathrm{Zn}^{2+}$ 形成了 $1: 1$ 的三元配合物, 由于 MAA 的 分子体积较小, 与模板分子的配位比为 $1: 1$ 且形成的 配合物较稳定有利于交联剂对复合物的固定, 洗脱除去 模板分子时留下较多的结合位点, 有助于对模板分子的 结合，但在 $\mathrm{NIP}_{1}$ 中同样存在较多的非选择性结合位点， 增大非选择性吸附的量. 故 $\mathrm{MIP}_{1}$ 及 $\mathrm{NIP}_{1}$ 的单点吸附量 均略高于 $\mathrm{MIP}_{2}$ 及 $\mathrm{NIP}_{2}$. 而 4-VP 的分子体积较大, 与模 板分子的配位比为 $1: 2$, 且形成的复合物的空间体积 较大, 有利于交联剂记忆固定三维结构, 在洗脱除去模 板分子后留下利于分子识别的特定的空间结构, 故 $\mathrm{MIP}_{2}$ 的印迹指数高于 $\mathrm{MIP}_{1}$.

\section{4 .2 混合溶液中聚合物的识别性能研究}

印迹聚合物的选择性吸附能力在混合溶液中能得 到很好地体现. 聚合物在含 $\mathrm{Zn}^{2+}$ 及不含有 $\mathrm{Zn}^{2+}$ 的混合 溶液中对 NFA 的吸附量分别为 $Q_{1}$ 和 $Q_{2}$; 聚合物在含 $\mathrm{Zn}^{2+}$ 及不含有 $\mathrm{Zn}^{2+}$ 的混合溶液中对 Ofloxacin 的吸附量 分别为 $Q_{3}$ 和 $Q_{4}$, 识别因子定义为: $K_{1}{ }^{\prime}=Q_{1} / Q_{3} ; K_{2}{ }^{\prime}=$ $Q_{2} / Q_{4}$. 由表 1 的结果显示, 印迹聚合物对底物溶液中的 NFA 及 Ofloxacin 均有吸附, 但对 NFA 的吸附量明显的 高于 Ofloxacin, $\mathrm{MIP}_{2}$ 对 NFA 的吸附量高于 $\mathrm{MIP}_{1}$ 但对 Ofloxacin 的吸附量低于 $\mathrm{MIP}_{1}$, 且 $K_{1}{ }^{\prime}$ 为 3.408 高于 $\mathrm{MIP}_{1}$, 说明 $\mathrm{MIP}_{2}$ 在混合溶液中的选择性优于 $\mathrm{MIP}_{1}$. 同时印迹 聚合物在含有 $\mathrm{Zn}^{2+}$ 溶液中对 NFA 的吸附量明显高于未 
加入 $\mathrm{Zn}^{2+}$ 溶液中的吸附量, 说明 $\mathrm{Zn}^{2+}$ 在选择性识别中 起到了桥梁及汼引作用, 在与 NFA 配位后并引领 NFA 进入印迹聚合物的特定的空间孔道中完成识别. 而 $K_{2}{ }^{\prime}$ 的值均略大于 1 说明印迹聚合物对 Ofloxacin 的识别为 非选择性的. 而表 1 中非印迹聚合物对底物的吸附量均 较小且其识别因子均接近于 1 , 即底物溶液中是否含有 金属离子对识别因子的影响较小, 可知非印迹聚合物对 两底物的吸附也是非选择性的.

表 1 混合溶液中印迹聚合物的特异性吸附量及识别因子

Table 1 Values of $Q$ and $K$ of polymers for mixed substrates

\begin{tabular}{ccccccc}
\hline Polymer & $Q_{1}{ }^{a}$ & $Q_{2}{ }^{b}$ & $Q_{3}{ }^{c}$ & $Q_{4}{ }^{d}$ & $K_{1}{ }^{\prime}$ & $K_{2}{ }^{\prime}$ \\
\hline $\mathrm{MIP}_{1}$ & 55.864 & 24.376 & 19.201 & 18.983 & 2.909 & 1.284 \\
$\mathrm{MIP}_{2}$ & 59.927 & 20.703 & 17.583 & 18.394 & 3.408 & 1.125 \\
$\mathrm{NIP}_{1}$ & 20.316 & 21.108 & 20.874 & 21.259 & 0.973 & 0.993 \\
$\mathrm{NIP}_{2}$ & 18.065 & 17.837 & 17.992 & 18.521 & 1.004 & 0.963 \\
\hline
\end{tabular}

${ }^{a}$ Capacity of the polymer for norfloxacin in the mixture solution with $\mathrm{Zn}^{2+}$;

${ }^{b}$ capacity of the polymer for norfloxacin in the mixture solution without $\mathrm{Zn}^{2+}$;

${ }^{c}$ capacity of the polymer for ofloxacin in the mixture solution with $\mathrm{Zn}^{2+}$;

${ }^{d}$ capacity of the polymer for ofloxacin in the mixture solution without $\mathrm{Zn}^{2+}$.

\section{3 结论}

沉淀聚合的方法成功地制备了诺氟沙星 $-\mathrm{Zn}^{2+}$ 分子 印迹聚合, 其中诺氟沙星、 $\mathrm{Zn}^{2+}$ 离子与两种功能单体分 别通过金属印迹方式形成了三元配合物, 通过对聚合物 性能的研究, 以碱性 4-乙烯基吡啶为功能单体制备的印 迹聚合物具有更好的识别性能, 且此金属印迹方式制备 的分子印迹聚合物可以用于氢键不易形成或易受干扰 的极性溶剂中进行分子识别, 识别环境更接近于天然分 子识别系统. 本文制备的金属分子印迹聚合物对诺氟沙 星具有良好的特异性识别能力, 在混合溶液中分离和识 别诺氟沙星具有良好的应用前景.

\section{4 实验部分}

\section{1 紫外分析}

\subsection{1 作用力的研究}

在加入 $0.5 \mathrm{~mL} 0.1 \mathrm{~mol} / \mathrm{L}$ 盐酸的乙腈溶液中固定 $\mathrm{NFA}$ 的浓度为 $0.025 \mathrm{mmol} / \mathrm{L}$, 配制 NFA 和 $\mathrm{Zn}^{2+}$ 的物质 的量比为 $1: 0,1: 0.5,1: 1$ 和 $1: 2$ 的系列溶液, 同时 配制 $n(\mathrm{NFA}): n\left(\mathrm{Zn}^{2+}\right): n$ (功能单体)为 $1: 1: 4$ 的溶液, 上述溶液震荡 $2 \mathrm{~h}$ 后在 $200 \sim 500 \mathrm{~nm}$ 波长范围内进行紫 外光谱扫描. 其中系列溶液的参比溶液为相应浓度的 $\mathrm{Zn}^{2}$ 溶液, 而加入功能单体样品的参比溶液为 $n(\mathrm{NFA}): n\left(\mathrm{Zn}^{2+}\right)=1: 1$ 溶液.

\subsection{2 配合比例的研究}

固定 $n(\mathrm{NFA}): n\left(\mathrm{Zn}^{2+}\right)=1: 1$ 的比例配制 NFA$\mathrm{Zn}^{2+}$ 与功能单体的物质的量比为 $1: 3,1: 5,1: 7,1: 9$ 和 $1: 12$ 的系列溶液上述溶液震荡 $2 \mathrm{~h}$ 后于紫外 $273 \mathrm{~nm}$
进行光度测量, 参比溶液为 $n(\mathrm{NFA}): n\left(\mathrm{Zn}^{2+}\right)=1: 1$ 溶 液.

\section{2 沉淀聚合法制备诺氟沙星 $-\mathrm{Zn}^{2+}$ 分子印迹聚合物}

称取一定量的模板分子与功能单体(表 2), 溶于含 有 $0.5 \mathrm{~mL} 0.1 \mathrm{~mol} / \mathrm{L}$ 盐酸的乙腈中 $(5 \mathrm{~mL})$, 置于冰箱中 过夜, 使模板分子与功能单体充分进行预组织并均匀的 分散在溶剂中. 后将预聚合溶液转入三口烧瓶中, 加入 $2 \mathrm{~mL}$ 乙二醇、 $6 \mathrm{~mL}$ EGDMA 及 $50 \mathrm{mg} \mathrm{AIBN}$ ，将混合液 超声 $5 \mathrm{~min}$ 后通氮气 $10 \mathrm{~min}$, 排除氧气, 氮气氛围下密 封并将其放入磁力搅拌器中先于 $20{ }^{\circ} \mathrm{C} 400 \mathrm{r} / \mathrm{min}$ 条件下 反应 $4 \mathrm{~h}$ 后升高温度至 $60{ }^{\circ} \mathrm{C} 400 \mathrm{r} / \mathrm{min}$ 搅拌 $8 \mathrm{~h}$. 反应完 成后, 以 $4000 \mathrm{r} / \mathrm{min}$ 离心分离 $10 \mathrm{~min}$, 移去上清液, 得 到分子印迹聚合物(MIP 系列). 后处理过程，将得到的 聚合物在索氏提取中以洗脱液甲醇-乙酸 $(8: 2$, 体积 比)的比例洗至无模板分子, 后将聚合物转移至 0.2 $\mathrm{mmol} / \mathrm{L}$ 的 EDTA 溶液中浸泡 $24 \mathrm{~h}$, 用以除去聚合物内 未完全反应的金属离子. 得到聚合物 $60{ }^{\circ} \mathrm{C}$ 干燥备用. 非印迹聚合物(NIP 系列)的制备方法同上.

表 2 聚合物的合成配比

Table 2 Composition of the imprinted polymer

\begin{tabular}{ccc}
\hline Polymer & Template $(n)$ & Monomer $(n)$ \\
\hline $\mathrm{MIP}_{1}$ & Norfloxacin-Zn $(1: 1)$ & $\operatorname{MAA}^{a}(4)$ \\
$\mathrm{MIP}_{2}$ & Norfloxacin-Zn $(1: 1)$ & $4-\mathrm{VP}^{b}(6)$ \\
$\mathrm{NIP}_{1}$ & - & $\operatorname{MAA}(4)$ \\
$\mathrm{NIP}_{2}$ & - & $4-\mathrm{VP}(6)$ \\
\hline
\end{tabular}

${ }^{a}$ Methacrylic acid, ${ }^{b} 4$-vinyl pyridine.

\section{3 红外光谱}

将诺氟沙星及印迹聚合物 $\mathrm{MIP}_{1}, \mathrm{MIP}_{2}$ 用 $\mathrm{KBr}$ 压片 法制样, 在 TENSOR37 型傅里叶变换红外光谱仪上扫 描各聚合物的红外谱图.

\section{4 扫描电镜分析}

将分子印迹聚合物 $\mathrm{MIP}_{2}$ 及 $\mathrm{NIP}_{2}$, 均匀黏贴在导电 胶上，镀金制样后，利用 QUANTA-200扫描电镜观察表 面形貌.

\section{5 聚合物的结合性能研究}

\subsection{1 等温吸附实验}

制备浓度为 $0.5 \sim 2.5 \mathrm{mmol} / \mathrm{L}$ 范围的 NFA- $\mathrm{Zn}^{2+}$ 含有 $0.5 \mathrm{~mL} 0.1 \mathrm{~mol} / \mathrm{L}$ 盐酸的乙腈溶液 $(50 \mathrm{~mL})$, 室温分别取 $\mathrm{MIP}_{1}, \mathrm{MIP}_{2}, \mathrm{NIP}_{1}$ 和 $\mathrm{NIP}_{2}(5 \mathrm{mg})$ 进行等温吸附实验吸附 时间为 $24 \mathrm{~h}$. 通过紫外测定溶液吸附前后的吸光度变 化. 根据公式 $Q=\left(C_{0}-C_{\mathrm{e}}\right) V / m$ 计算吸附量, 取 3 次平行 测定结果. 式中: $Q$ 为底物结合量 $(\mu \mathrm{mol} / \mathrm{g}), C_{0}$ 为底物的 初始浓度 $(\mathrm{mmol} / \mathrm{L}), C_{\mathrm{e}}$ 为吸附后底物的平衡浓度 $(\mathrm{mmol} / \mathrm{L}), V$ 为溶液的体积 $(\mathrm{mL}), m$ 为 MIP 的质量 $(\mathrm{g})$.

\subsection{2 混合溶液中选择性识别实验}

分别取 $\mathrm{MIP}_{1}, \mathrm{MIP}_{2}, \mathrm{NIP}_{1}$ 和 $\mathrm{NIP}_{2}(5 \mathrm{mg})$ 加入 
$n(\mathrm{NFA}): n\left(\mathrm{Zn}^{2+}\right)=1: 1$ 及 $n($ Ofloxacin $): n\left(\mathrm{Zn}^{2+}\right)=1: 1$ 的混合溶液 $(2.5 \mathrm{mmol} / \mathrm{L}, 50 \mathrm{~mL})$, 再称取等量的 $\mathrm{MIP}_{1}$ 和 $\mathrm{MIP}_{2}$ 并将底物换做含有 NFA 和 Ofloxacin 的同浓度乙腈 溶液, 进行等温吸附实验吸附时间为 $24 \mathrm{~h}$, 按上述方法 计算吸附量.

\section{References}

[1] Alexander, C.; Davidson, L.; Hayes, W. Tetrahedron 2003, 59(12), 2025.

[2] Alexander, C.; Andersson, H. S.; Andersson, L. I.; Ansell, R. J.; Kirsch, N.; Nicholls, I. A.; O'Mahony, J.; Whitcombe, M. J. J. Mol. Recognit. 2006, 19(2), 106.

[3] Masque, N.; Marce, R. M.; Borrull, F. TrAC, Trends Anal. Chem. 2001, 20(9), 477.

[4] Apodaca, D. C.; Pernites, R. B.; Ponnapati, R.; Del Mundo, F. R.; Advincula, R. C. Macromolecules 2011, 44(17), 6669.

[5] Chen, Y. X.; Gao, B. J.; Jiang, G. M.; Zhang, R. X. Acta Chim. Sinica 2011, 69(14), 1705. (陈迎金金, 高保娇, 姜桂明, 张瑞霞, 化 学学报, 2011, 69(14), 1705.)

[6] Gupta, R.; Kumar, A. J. Sol-Gel Sci. Technol. 2011, 58(1), 182.

[7] Li, D.; Gao, B. J.; Xu, W. M. Acta Chim. Sinica 2011, 69(24), 3019. (李丁, 高保娇, 许文梅, 化学学报, 2011, 69(24), 3019.)

[8] Chen, L. X.; Xu, S. F.; Li, J. H. Chem. Soc. Rev. 2011, 40(5), 2922.

[9] Vasapollo, G.; Del Sole, R.; Mergola, L.; Lazzoi, M. R.; Scardino, A.; Scorrano, S.; Mele, G. Int. J. Mol. Sci. 2011, 12(9), 5908.

[10] Long, Y. Y.; Philip, J. Y. N.; Schillen, K.; Liu, F.; Ye, L. J. Mol. Recognit. 2011, 24(4), 619.

[11] Zhu, G. F.; Gao, Y. B.; Gao, X.; Fan, J. Acta Chim. Sinica 2011,
69(8), 973. (朱桂芬, 高燕哺, 高霞, 樊静, 化学学报, 2011, 69(8), 973.)

[12] Mohajeri, S. A.; Karimi, G.; Aghamohammadian, J.; Khansari, M. R. J. Appl. Polym. Sci. 2011, 121(6), 3590.

[13] Akperov, O. H.; Maharramov, A. M.; Akperov, E. O.; Kadimova, H. A. Iran. Polym. J. 2010, 19(9), 717.

[14] Bagheri, B.; Abdouss, M.; Aslzadeh, M. M.; Shoushtari, A. M. Iran. Polym. J. 2010, 19(12), 911.

[15] Wang, X. H.; Chen, L. R.; Xu, X. J.; Li, Y. Z. Anal. Bioanal. Chem. 2011, 401(4), 1423

[16] Yang, W. H.; Yan, S. L.; Wei, C.; Wang, Q. Z. Acta Polym. Sinica 2010, (10)，1163. (杨卫海，严守雷，卫晨，王清章，高分子学报, 2010, (10), 1163.)

[17] Azimi, O.; Salari, H.; Emami, Z.; Chamani, J. Eur. Biophys. J. Biophys. Lett. 2011, 40, 38.

[18] Bel'tyukova, S. V.; Malinka, E. V.; Liventsova, E. O. J. Water Chem. Technol. 2008, 30(1), 38

[19] Conkle, J. L.; Lattao, C. V.; White, J. R.; Cook, R. L. Anal. Lett. 2009, 42(18), 2937.

[20] Khallow, K. I.; Al-Assaf, A. Y. R. E-Journal of Chemistry 2011, $8(2), 576$.

[21] Refat, M. S.; Mohamed, G. G.; de Farias, R. F.; Powell, A. K.; El-Garib, M. S.; El-Korashy, S. A.; Hussien, M. A. J. Therm. Anal. Calorim. 2010, 102(1), 225.

[22] Gautam, L.; Scott, K. S.; Cole, M. D. J. Anal. Toxicol. 2005, 29(5), 339.

[23] Elghobashi, N.; Gonzalez, L.; Manz, J. Zeitschrift Fur Physikalische Chemie-International Journal of Research in Physical Chemistry \& Chemical Physics 2003, 217(12), 1577.

[24] Zhang, M. S.; Huang, J. R.; Tang, L. P. Acta Chim. Sinica 2009, 67(24), 2840. (张茂升, 黄佳蓉, 唐丽萍, 化学学报, 2009, 67(24), 2840.) 